\title{
Genetic Polymorphisms Associated with Age-Related Macular Degeneration
}

\author{
Zulvikar Syambani Ulhaq ${ }^{1}$ \\ ${ }^{1}$ Department of Biomedical Science, Faculty of Medicine and Health Sciences, Universitas Islam Negeri \\ Maulana Malik Ibrahim Malang, Indonesia
}

\author{
*Correspondence to: \\ Zulvikar Syambani Ulhaq \\ zulhaq@kedokteran.uin-malang.ac.id, \\ Universitas Islam Negeri Maulana Malik \\ Ibrahim Malang, Indonesia
}

\begin{abstract}
Age-related macular degeneration (AMD) is one of the leading causes of irreversible blindness in older individuals. AMD is often classified into two clinical categories: non-neovascular AMD (nonexudative/dry AMD) and neovascular AMD (exudative/wet AMD).
\end{abstract}

Several studies provide evidence that genetic polymorphisms have been implicated to influence the development and progression of AMD. Moreover, a report regarding a meta-analysis of specific gene polymorphism with AMD becomes popular in the last 5 years. Using online search databases with keywords including "polymorphism", "age-related macular degeneration", and "meta-analysis", I summarized the findings from the latest published meta-analysis ${ }^{1-18}$ as shown in Figure 1. Moreover, in this short communication additional meta-analysis of TNFa, CCL-2, MMP3, MMP7, MMP9, and TIMP2 were also included $^{19-29}$.

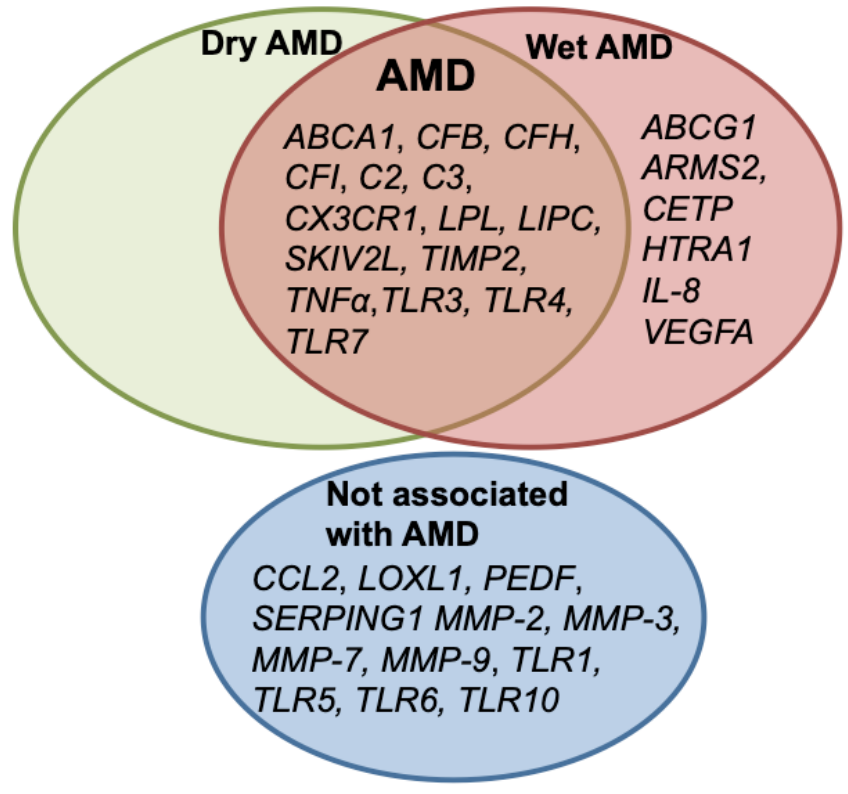

Figure 1. Summary of genetic polymorphisms associated with AMD.

It was shown that a protective effect of TNF $\alpha-863 \mathrm{~A}$ carrier ( $\mathrm{A}$ vs $\mathrm{C}, \mathrm{OR}=0.67, \mathrm{P}=0.007 ; \mathrm{AA}+\mathrm{CA}$ vs $C \mathrm{C}, \mathrm{OR}=$ $0.58, \mathrm{P}=0.001$; $C A$ vs $A A+C C, O R=0.55, P=0.001 ;$ and $C A$ vs $C C, O R=0.54, P=0.001$, Figure 2A-D) and TIMP2 $-418 \mathrm{C}$ carrier ( $\mathrm{GC}$ vs $\mathrm{CC}+\mathrm{GG}, \mathrm{OR}=0.54, \mathrm{P}=0.018$; and $\mathrm{GC}$ vs $\mathrm{GG}, \mathrm{OR}=0.56, \mathrm{P}=0.046$, Figure $2 \mathrm{E}, \mathrm{F}$ ) to the development of AMD. No association was observed in polymorphism of TNFa -1031 T/C, TNFa -308 G/A, TNFa -238 G/A, CCL-2 -2158 A/G, MMP3 -1771 5A/6A, MMP7 -181 A/G, MMP9 -1562 C/T with 
AMD (data not shown). Analysis of IL-8/CXCL8 $+781 C / T$ polymorphism indicated that the TT genotype is associated with the risk of AMD (in submission). Together with previously published articles, twenty-one genes were analyzed for gene network and gene ontology analysis. Based on gene ontology (GO) analysis, fifty-five clusters were identified, among them regulation of response to stress/external stimuli and immune/inflammatory responses were strongly correlated with AMD (Figure $\mathbf{3}$ ). In summary, this report emphasizes that neuroinflammation plays a pivotal role in the development of AMD.

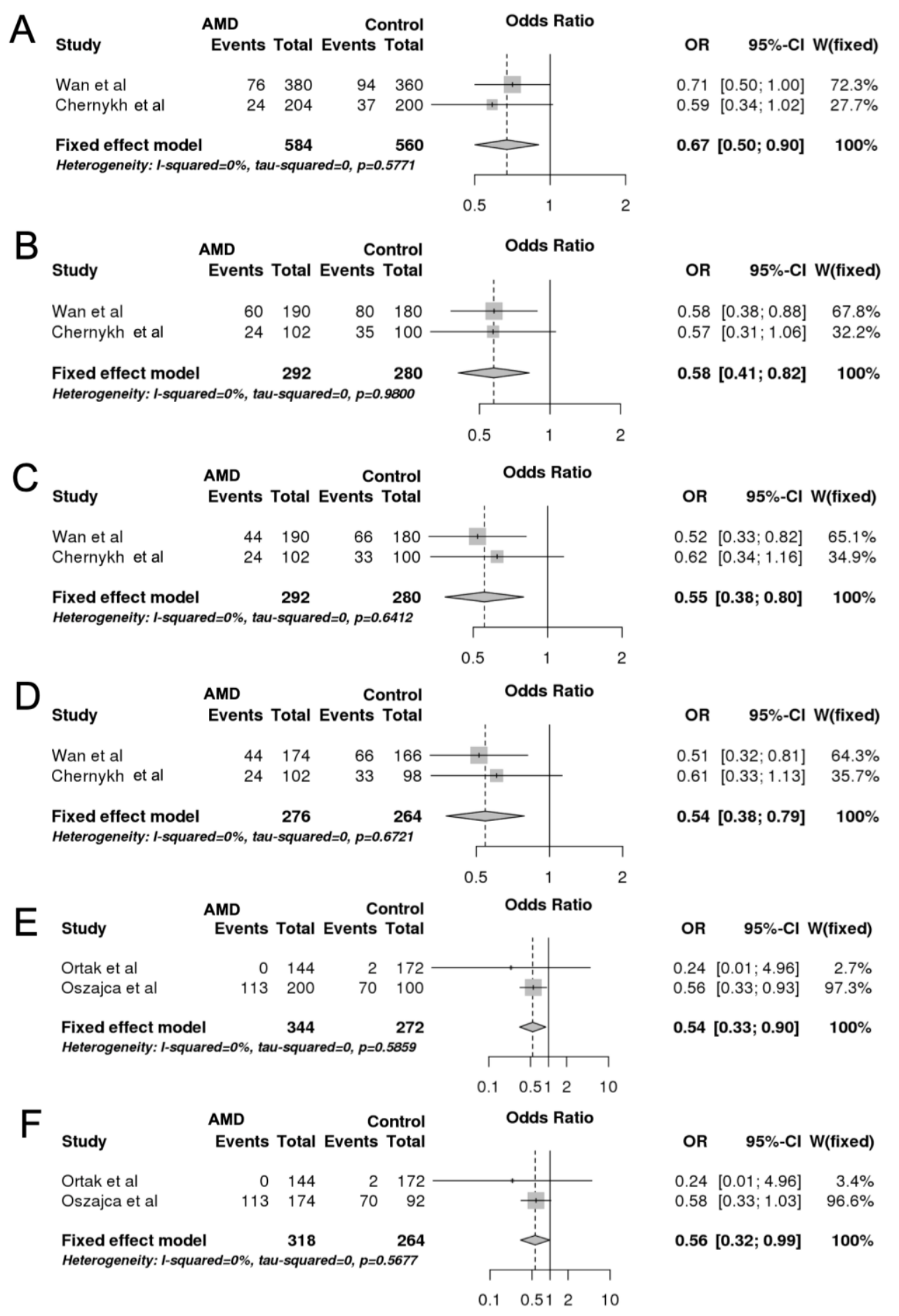

Figure 2. Association of TNFa -863 C/A and TIMP2 -418 G/C polymorphisms with AMD. (A) Allelic model in TNFa -863 C/A; (B) Dominant model in TNFa -863 C/A; (C) Overdominant model in TNFa -863 C/A; (D). Heterozygous model in TNF $\alpha-863$ C/A; (E) Overdominant model in TIMP2 -418 G/C; (F). Heterozygous model in TIMP2 -418 G/C. 


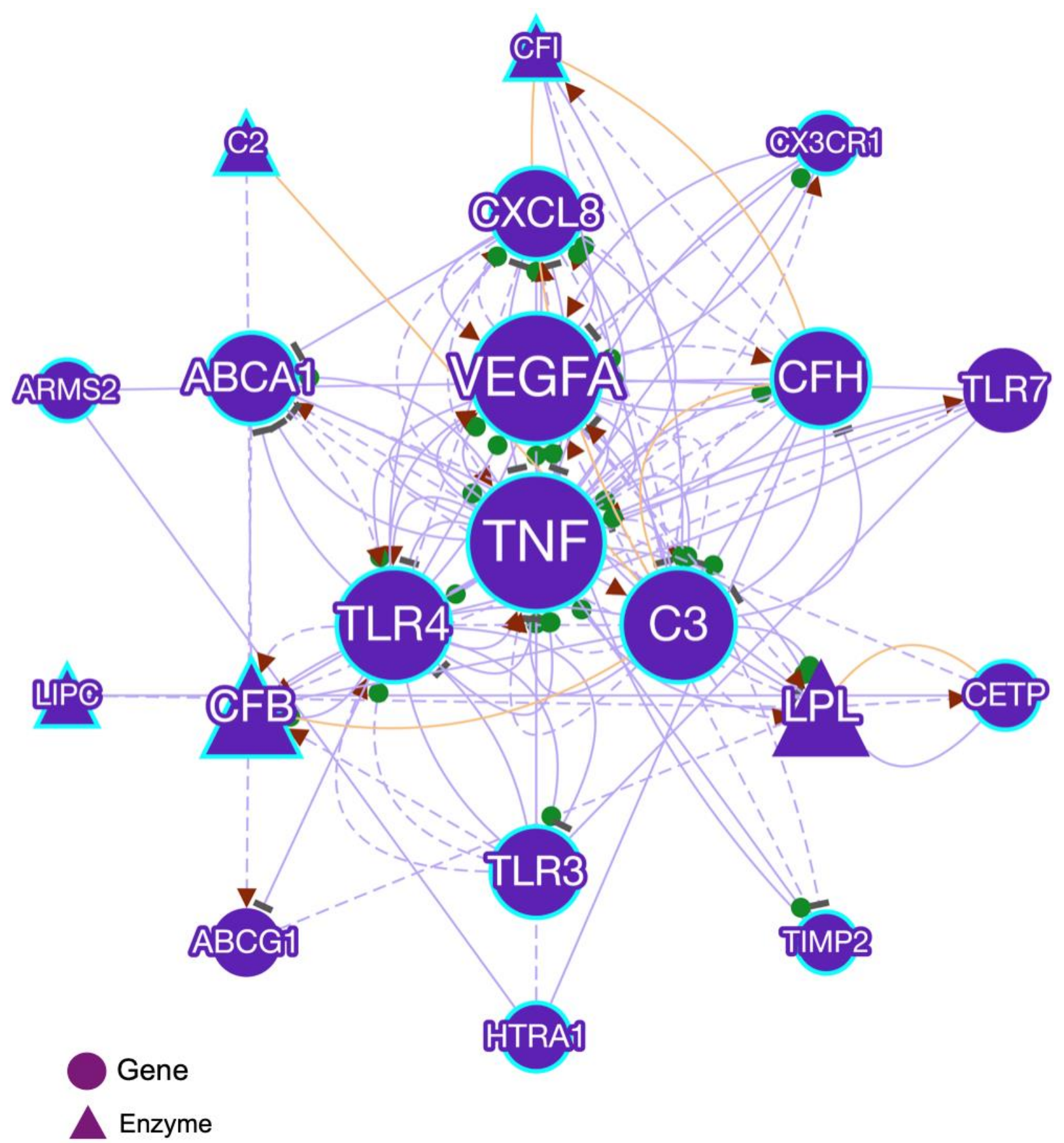

Figure 3. Gene network analysis. The size of the node represents the number of connections in this network. This result implies that immune and inflammatory responses as a central mechanism associated with AMD.

\section{Abbreviations}

ABCA1: ATP binding cassette subfamily A member 1

ABCG1: ATP binding cassette subfamily $\mathrm{G}$ member 1

ARMS2: age-related maculopathy susceptibility 2

AMD: age-related macular degeneration

$\mathrm{C} 2 / 3$ : complement $\mathrm{C} 2 / 3$

CCL2: C-C motif chemokine ligand 2

CETP: cholesteryl ester transfer protein

CFB: complement factor B

$\mathrm{CFH}$ : complement factor $\mathrm{H}$

CFI: complement factor I 
CX3CR1: C-X3-C motif chemokine receptor 1

CXCL8/IL-8: C-X-C motif chemokine ligand 8/Interleukin-8

HTRA1: HtrA serine peptidase 1

LIPC: lipase $C$, hepatic type

LOXL1: lysyl oxidase like 1

LPL: lipoprotein lipase

PEDF: Pigment epithelium-derived factor

MMP2/3/7/9: matrix metallopeptidase 2/3/7/9

SERPING1: serpin family $\mathrm{G}$ member 1

SKIV2L: Ski2 like RNA helicase

TIMP2: TIMP metallopeptidase inhibitor 2

TLR1/3/4/6/7/9/10: toll like receptor 1/3/4/6/7/9/10

TNF: tumor necrosis factor

VEGFA: vascular endothelial growth factor A

\section{REFERENCES}

1. Usategui-Martín R, Pastor-Idoate S, Chamorro AJ, Fernández I, Fernández-Bueno I, Marcos-Martín M, et al. Meta-analysis of the rs243865 MMP-2 polymorphism and age-related macular degeneration risk. PLoS One 2019;14(3):e0213624. doi: 10.1371/journal.pone.0213624.

2. Jabbarpoor Bonyadi $\mathrm{MH}$, Yaseri $M$, Nikkhah $H$, Bonyadi $M$, Nazari $R$, Soheilian $M$. Comparison of ARMS2/LOC387715 A69S and CFH Y402H risk effect in wet-type age-related macular degeneration: a meta-analysis. Int Ophthalmol 2019;39(4):949-956. doi: 10.1007/s10792-018-0853-y.

3. Su Y, Hu Z, Pan T, Chen L, Xie P, Liu Q Complement factor B gene polymorphisms and risk of age-related macular degeneration: A meta-analysis. Eur J Ophthalmol. 2019:1120672119840245. doi: $10.1177 / 1120672119840245$.

4. Wang Q, Zhao HS, Li L. Association between complement factor I gene polymorphisms and the risk of age-related macular degeneration: a Meta-analysis of literature. Int J Ophthalmol 2016;9(2):298-305. doi: 10.18240/ijo.2016.02.23.

5. Zhang J, Li S, Hu S, Yu J, Xiang Y. Association between genetic variation of complement C3 and the susceptibility to advanced age-related macular degeneration: a meta-analysis. BMC Ophthalmol 2018;18(1):274. doi: 10.1186/s12886-018-0945-5.

6. Lu F, Liu S, Hao Q, Liu L, Zhang J, Chen X, et al. Association Between Complement Factor C2/C3/CFB/CFH Polymorphisms and Age-Related Macular Degeneration: A Meta-Analysis. Genet Test Mol Biomarkers 2018;22(9):526-540. doi: 10.1089/gtmb.2018.0110.

7. Yuan MZ, Han RA, Zhang CX, Chen YX. Association of Genes in the High-Density Lipoprotein Metabolic Pathway with Polypoidal Choroidal Vasculopathy in Asian Population: A Systematic Review and MetaAnalysis. J Ophthalmol 2018;2018:9538671. doi: 10.1155/2018/9538671.

8. Shuai P, Ye Z, Liu Y, Qu C, Liu X, Luo H, et al. Association between SKIV2L polymorphism rs429608 and age-related macular degeneration: A meta-analysis. Ophthalmic Genet 2017;38(3):245-251. doi: 10.1080/13816810.2016.1210650.

9. Li D, Peng X, Sun H. Association of CX3CR1 (V249I and T280M) polymorphisms with age-related macular degeneration: a meta-analysis. Can J Ophthalmol 2015;50(6):451-60. doi: 10.1016/j.jcjo.2015.08.010.

10. Wang Y, Wang M, Han Y, Zhang R, Ma L. ABCA1 rs1883025 polymorphism and risk of age-related macular degeneration. Graefes Arch Clin Exp Ophthalmol 2016;254(2):323-32. doi: 10.1007/s00417-015-3211-z.

11. Wang YF, Han Y, Zhang R, Qin L, Wang MX, Ma L. CETP/LPL/LIPC gene polymorphisms and susceptibility to age-related macular degeneration. Sci Rep 2015;5:15711. doi: 10.1038/srep15711. 
12. Barchitta M, Maugeri A. Association between Vascular Endothelial Growth Factor Polymorphisms and Age-Related Macular Degeneration: An Updated Meta-Analysis. Dis Markers 2016;2016:8486406. doi: $10.1155 / 2016 / 8486406$.

13. Ma L, Tang SM, Rong SS, Chen H, Young AL, Kumaramanickavel G, et al. Association of PEDF polymorphisms with age-related macular degeneration and polypoidal choroidal vasculopathy: a systematic review and meta-analysis. Sci Rep 2015;5:9497. doi: 10.1038/srep09497.

14. Dong $\quad \mathrm{Y}, \mathrm{Li} \quad \mathrm{ZD}$, Fang $\mathrm{XY}$, Shi XF, Chen $\mathrm{S}$, Tang $\mathrm{X}$. Association between SERPING1 rs2511989 polymorphism and age-related macular degeneration: Meta-analysis. Int J Ophthalmol 2015;8(2):385-94. doi: 10.3980/j.issn.2222-3959.2015.02.31.

15. Ma L, Tang FY, Chu WK, Young AL, Brelen ME, Pang CP, et al. Association of toll-like receptor 3 polymorphism rs3775291 with age-related macular degeneration: a systematic review and metaanalysis. Sci Rep 2016;6:19718. doi: 10.1038/srep19718.

16. Ma L, Li Z, Liu K, Rong SS, Brelen ME, Young AL, et al. Association of Genetic Variants with Polypoidal Choroidal Vasculopathy: A Systematic Review and Updated Meta-analysis. Ophthalmology 2015;122(9):1854-65. doi: 10.1016/j.ophtha.2015.05.012.

17. Xiying M, Wenbo W, Wangyi F, Qinghuai L. Association of Apolipoprotein E Polymorphisms with Agerelated Macular Degeneration Subtypes: An Updated Systematic Review and Meta-analysis. Arch Med Res 2017;48(4):370-377. doi: 10.1016/j.arcmed.2017.08.002.

18. Edwards AO, Chen D, Fridley BL, James KM, Wu Y, Abecasis G, et al. Toll-like receptor polymorphisms and age-related macular degeneration. Invest Ophthalmol Vis Sci 2008;49(4):1652-9. doi: 10.1167/iovs.07-1378.

19. Liutkevičienė $R$, Žaliaduonytè-Pekšienė $D$, Žaliūnienè $D$, Gustienè $O$, Jašinskas $V$, Lesauskaitè $V$, et al. Does matrix metalloproteinase-3 polymorphism play a role in age-related macular degeneration in patients with myocardial infarction? Medicina (Kaunas) 2012;48(8):404-9.

20. Liutkeviciene $\quad R$, Lesauskaite $\quad$, Sinkunaite-Marsalkiene $\quad G$, Zaliuniene $\quad D$, Zaliaduonyte-Peksiene $D$, Mizariene V, et al. The Role of Matrix Metalloproteinases Polymorphisms in Age-Related Macular Degeneration. Ophthalmic Genet 2015;36(2):149-55. doi: 10.3109/13816810.2013.838274.

21. Budiene B, Liutkeviciene R, Gustiene O, Ugenskiene R, Laukaitiene D, Savukaityte A, et al. The association of matrix metalloproteinases polymorphisms and interleukins in advanced age-related macular degeneration. Ophthalmic Genet 2018;39(4):463-472. doi: 10.1080/13816810.2018.1484928.

22. Oszajca K, Szemraj M, Szemraj J, Jurowski P. Association analysis of genetic polymorphisms and expression levels of selected genes involved in extracellular matrix turnover and angiogenesis with the risk of age-related macular degeneration. Ophthalmic Genet 2018;39(6):684-698. doi: 10.1080/13816810.2018.1525752.

23. Ortak H, Demir S, Ateş Ö, Benli I, Sögüt E, Sahin M. The role of MMP2 (-1306C>T) and TIMP2 (-418 G>C) promoter variants in age-related macular degeneration. Ophthalmic Genet 2013;34(4):217-22. doi: 10.3109/13816810.2013.781192.

24. Sharma NK, Sharma K, Singh R, Sharma SK, Anand A. CCL2 single nucleotide polymorphism of rs 1024611 implicates prominence of inflammatory cascade by univariate modeling in Indian AMD. PLoS One 2018;13(4):e0193423. doi: 10.1371/journal.pone.0193423.

25. Jabbarpoor Bonyadi MH, Mohammadian T, Bonyadi M, Soheilian M, Moein H, Yaseri M. Evaluation of Creactive protein and CC-cytokine ligand 2 polymorphism interaction for age-related macular degeneration. Ophthalmic Genet 2016;37(4):465-467. doi: 10.3109/13816810.2015.1120317.

26. Bonyadi M, Foruzandeh Z, Mohammadian T, Fotouhi N, Soheilian M, Jabbarpoor Bonyadi $\mathrm{MH}$, et al. Evaluation of CC-cytokine ligand 2 and complementary factor $\mathrm{H} \mathrm{Y402H} \mathrm{polymorphisms} \mathrm{and} \mathrm{their}$ interactional association with age-related macular degeneration. Acta Ophthalmol 2016;94(8):e779e785. doi: 10.1111/aos.13143.

27. Wan L, Lin HJ, Tsai Y, Lee CC, Tsai CH, Tsai FJ, et al. Tumor necrosis factor- $\alpha$ gene polymorphisms in agerelated macular degeneration. Retina 2010;30(10):1595-600. doi: 10.1097/IAE.0b013e3181dc58a6. 
28. Bonyadi MH, Bonyadi M, Ahmadieh H, Fotuhi N, Shoeibi N, Saadat S, et al. Tumor Necrosis Factor Gene Polymorphisms in Advanced Non-exudative Age-related Macular Degeneration. J Ophthalmic Vis Res 2015;10(2):155-9. doi: 10.4103/2008-322X.163781.

29. Chernykh V, Shevchenko A, Konenkov V, Prokofiev V, Eremina A, Trunov A. TNF- $\alpha$ gene polymorphisms: association with age-related macular degeneration in Russian population. Int $\mathrm{J}$ Ophthalmol 2019;12(1):25-29. doi: 10.18240/ijo.2019.01.04. 